\title{
Research
}

Leone Ridsdale, Judith Charlton, Mark Ashworth, Mark P Richardson and Martin C Gulliford

\section{Epilepsy mortality and risk factors for death in epilepsy:}

\author{
a population-based study
}

\begin{abstract}
Background

Epilepsy is an important cause of amenable mortality but risk factors for death in epilepsy are not well understood.

\section{Aim}

To evaluate trends in epilepsy mortality in a large population and identify risk factors for death in epilepsy.

\section{Design and setting}

Nested case-control study in the UK, using data from the General Practice Research Database (GPRD) from 1993 to 2007

\section{Method}

Participants were included if they had ever been diagnosed with epilepsy and prescribed anticonvulsant drugs. Trends in all-cause mortality in persons with epilepsy in the GPRD were compared with death registrations with epilepsy as the underlying cause. A nested case-control study was implemented to compare participants with epilepsy who died with those who did not die.

\section{Results}

The prevalence of epilepsy increased from 9 per 1000 in 1993 to 12 per 1000 in 2007, and epilepsy deaths also increased in this period. In a nested case-control study, mortality was associated with: recorded alcohol problems lodds ratio [OR] $2.96,95 \%$ confidence interval $[\mathrm{Cl}]=2.25$ to 3.89 , $P<0.001)$. having collected the last anticonvulsant prescription $90-182$ days previously (OR 1.83, $\mathrm{Cl}=1.66$ to $2.03, P<0.001$ ): having an injury in the previous year (OR 1.41 . $95 \% \mathrm{Cl}=1.30$ to $1.53, P<0.001$ ), and having been treated for depression (OR $1.39,95 \% \mathrm{Cl}=1.28$ to 1.50, $P<0.001$ ). In data available from 2004 onwards, being recorded seizure free in the previous 12 months was associated with lower mortality (OR 0.78, $95 \% \mathrm{Cl}=0.71$ to 0.86 ,

\section{INTRODUCTION}

Deaths that are amenable to medical intervention through risk factors such as hypertension, or preventable by individual behaviour change or public health measures like smoking, have been targeted in the UK's NHS policies.' Mortality from all causes in the general population of England and Wales declined by $16 \%$ between 1993 and 2005; in contrast, mortality with epilepsy recorded as an underlying cause increased by $31 \%$ in males and $39 \%$ in females during this period. ${ }^{2}$

Epilepsy is ranked as the fifth highest amenable cause of years of life lost before the age of 75 for males, and eighth highest for females. ${ }^{3}$ A cohort study of people with epilepsy dying over an 8-year period reported that $30 \%$ of those died of accidents (mostly drowning and burns), $23 \%$ died suddenly, $16 \%$ died in status epilepticus, and $14 \%$ committed suicide. ${ }^{4}$ An audit of sudden epilepsy-related deaths in the UK found a lack of communication between professionals and with families, and estimated that $40 \%$ of adult and $60 \%$ of child deaths were potentially avoidable through improved care. ${ }^{5}$

In 2004, the NHS introduced a new system of criteria-linked activity and reward for primary care, called the Quality and Outcomes Framework (QOF). ${ }^{6}$ For the first time, services for patients with epilepsy were financially rewarded in primary care
\end{abstract} $P<0.001)$

\section{Conclusion}

Mortality with epilepsy appears to be increasing. Patients who have alcoho problems, do not collect repeat prescriptions for anticonvulsant drugs, have recent injuries, or have been treated for depression may be at increased risk of death; patients who remain seizure free over 12 months are at a lower risk.

\section{Keywords}

cohort study; epilepsy mortality; epilepsy prevalence; nested case-control study primary care.
MP Richardson, FRCP, Paul Getty III, professor of epilepsy, Department of Clinical Neuroscience; L Ridsdale, FRCP, professor of primary care M Ashworth, MRCGP, senior lecturer in general practice; MC Gulliford, FFPH, professor of public health, Department of Primary Care and Public Health Sciences, King's College London, London.

\section{Address for correspondence}

Martin Gulliford, Department of Primary Care and Public Health Sciences, King's College London, neurology; J Charlton, MSc, research assistant;
- the criteria being maintaining registers of patients with epilepsy, conducting an annual review, and recording seizure frequency. There remained a lack of epidemiological evidence to underpin new strategies designed to improve epilepsy self-care and reduce mortality in epilepsy.

The present study aimed to identify risks for death in epilepsy in a large population. Data were obtained from the UK General Practice Research Database (GPRD) to conduct a cohort study of people with epilepsy in order to evaluate long-term trends in epilepsy including incidence, prevalence, and mortality. Mortality was evaluated before and after the introduction of the QOF. Using prior evidence, it was hypothesised that social and material deprivation, recent discontinuation of anticonvulsant drugs, treatment for depression, a history of injuries, and a failure to remain seizure free might be associated with mortality in epilepsy. ${ }^{7}$ A nested case-control study was implemented to test these hypotheses.

\section{METHOD}

The protocol for the study was approved by the Independent Scientific Advisory Committee for Medicines and Healthcare products Regulatory Agency (MHRA) database research (Protocol 09-006). The GPRD includes records from approximately 1990 to the present day; these data are subject to quality checks and are referred to

7th Floor, Capital House, 42 Weston Street London SE1 3QD.

E-mail: martin.gullifordakcl.ac.uk

Submitted: 1 October 2010; Editor's response: 19 November 2010; final acceptance: 6 December 2010

\section{British Journal of General Practice}

This is the full-length article lpublished online 26 Apr 2011) of an abridged version published in print. Cite this article as: Br J Gen Pract 2011; DOI: 10.3399/bjgp11X572463. 


\section{How this fits in}

Although mortality from all causes is declining rapidly in the general population, this study shows that mortality from epilepsy may be increasing. Death in epilepsy is more likely when seizures have continued to occur, where there are alcohol problems, when anticonvulsant prescriptions are not renewed, where injury has occurred in the previous year, or when patients have been treated for depression. Primary care professionals should be alert to the presence of these risk factors in order to step up care for people with epilepsy who are at increased risk of death.

as 'up to standard' when they are of a quality that is high enough to be used in research. Several studies have evaluated the validity of clinical diagnoses recorded in the GPRD with, generally, satisfactory results. Herrett et al found that the median proportion of GPRD cases with a confirmed diagnosis in validation studies was $89 \% .{ }^{8}$

For this study, participants who had been diagnosed with epilepsy at some point in their life and had received one or more prescriptions for anticonvulsant drugs were selected. Medical diagnoses for epilepsy were identified using a list of 186 Read and Oxford Medical Information Systems Codes for epilepsy; details of the codes are available from the authors. Anticonvulsant prescriptions were identified using multilex codes for drugs included in section 4.8.1 of the British National Formulary. ${ }^{9}$ These included beclamide, carbamazepine, clobazam, ethosuximide, fospheytoin, gabapentin, lacosamide, lamotrigine, levetiracetam, mesuximide, methylphenobarbital, oxcarbazepine, phenobarbital, phenytoin, pregabalin, primidone, rufinamide, sodium valproate, stiripentol, sultiame, tiagibine, topiramate, valproic acid, vigabatrin and zonisamide. Benzodiazepine drugs were not included as most prescriptions are likely to be for anxiety or insomnia rather than epilepsy.

For this study, a participant was considered to have prevalent epilepsy if:

- epilepsy was ever recorded in their medical record; and

- if one or more prescriptions for anticonvulsant drugs were recorded since the date of their registration at the practice or the date on which the practice entered the GPRD if this was later.

Participants were considered to have 'incident epilepsy' if the index date - the earlier of the first medical code or the first anticonvulsant prescription - was more than 24 months after the start date. Mortality from all causes in participants with prevalent epilepsy was derived from the death records in the GPRD; causespecific mortality was not available for analysis. Age-standardised rates were estimated using the European Standard Population for reference.

A nested case-control study was implemented. Participants with prevalent epilepsy who died from any cause between 1993 and 2006 formed the cases; for each of these, two controls were randomly selected from the set of all participants with prevalent epilepsy who were alive at the date of death. Each control was chosen to be at risk' throughout the risk period of the matched case. Cases and controls were also matched for sex and year of birth, and compared with regard to the following explanatory variables. The deprivation score of the practice was obtained based on the general practice's postcode. Practices were divided into quintiles based on the practice Indices of Multiple Deprivation 2007 score; each deprivation quintile was retained in the final model because deprivation was believed to be a relevant potential predictor of epilepsy mortality.

The medical records of cases and controls were evaluated in the 12 months before the formers' death date for recorded problems of:

- alcohol misuse;

- whether the participants were ever diagnosed with depression and treated with antidepressant drugs in the preceding 12 months;

- whether there was any history of injury (including accidents, fractures, or burns) in the preceding 12 months; and

- whether participants were recorded as seizure free in the preceding 12 months using a single Read Code (667F) that was introduced in association with the QOF in 2004. 


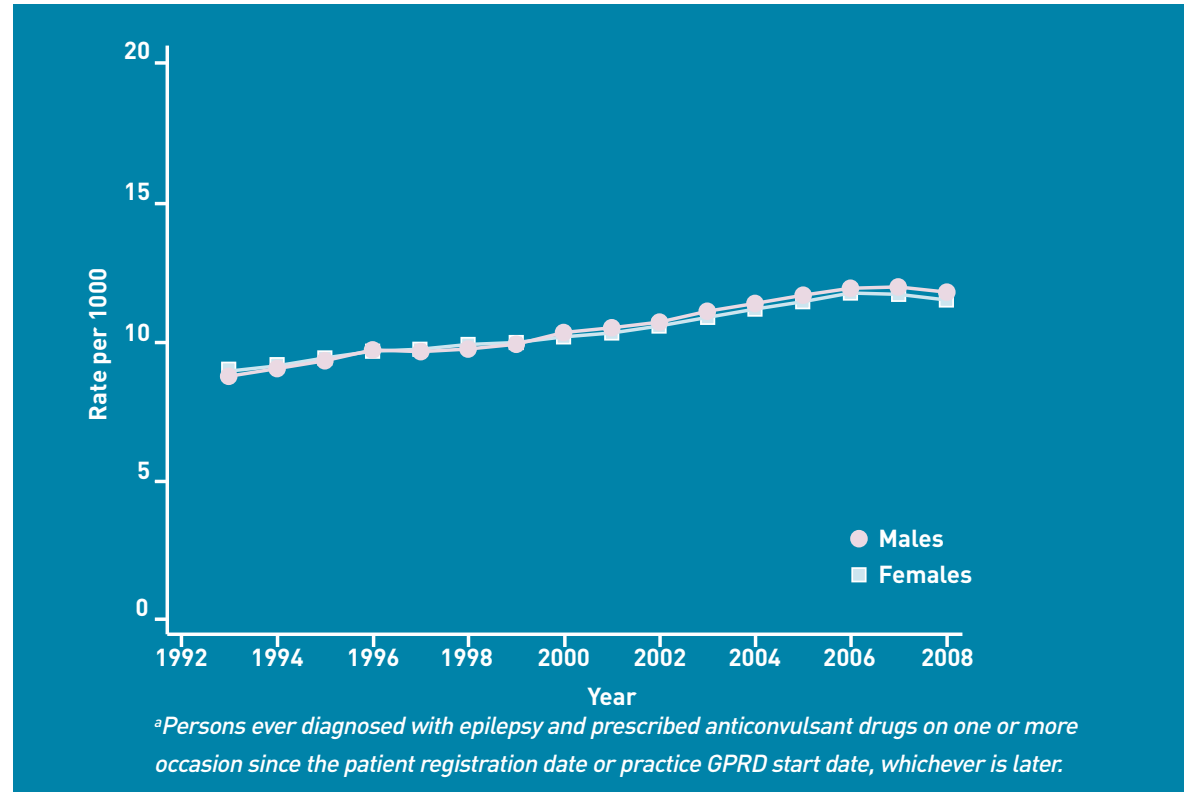

Figure 1. Prevalence of epilepsy in the General Practice Research Database (GPRD) (1993-2007.)

Figure 2. Mortality from all causes (1992-2007) in persons ever diagnosed with epilepsy in the General Practice Research Database. ${ }^{a}$

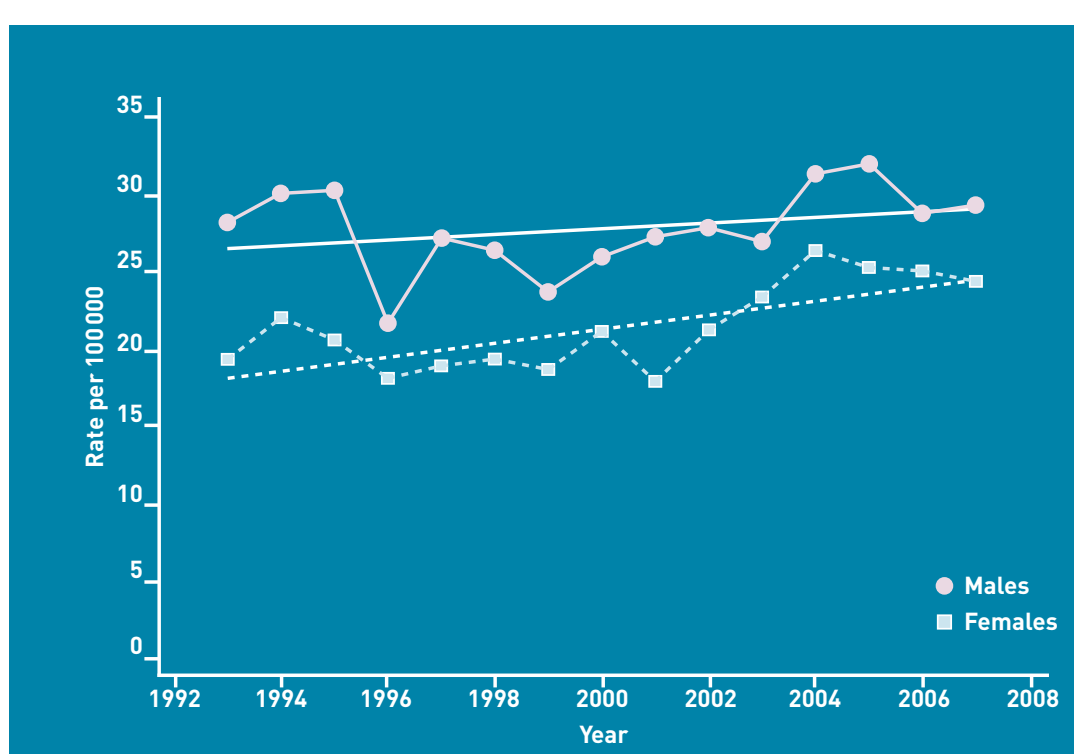

${ }^{a}$ Rate given is per 100000 patients registered on the General Practice Research Database. the European Standard Population for reference. Linear trends were estimated from a linear regression of agestandardised rate on year. Mortality statistics were coded according to the ninth revision of the International Classification of Diseases (ICD-9) ${ }^{10}$ from 1979 to 1999, and according to the tenth revision (ICD-10)1 from 2000 onwards. Analyses from the ONS suggest comparability ratios (ICD-10 to ICD-9) of 1.014 for males and 1.233 for females; a sensitivity analysis was, therefore, implemented in which numbers of deaths were adjusted for comparability between ICD-9 and ICD-10.

\section{RESULTS}

There were 434 general practices included in the study, and a total of 1.5 million registered patients in 1993, which increased to 3.3 million in 2007. The agestandardised incidence of new epilepsy diagnoses was approximately 34 per 100000 per year in females and 39 per 100000 per year in males, with weak evidence for a slight decline during the period of study. Figure 1 shows the prevalence of epilepsy in the GPRD by year and sex. From 1993 to 2007, the prevalence of epilepsy increased from about $0.9 \%$ to about $1.2 \%$ in both males and females. In a linear regression model, there was strong evidence of an increasing linear trend of about $0.2 \%$ per decade in both males and females.

Figure 2 shows mortality by year of study; Table 1 shows the mortality from all causes of persons with prevalent epilepsy by 3-year period from 1993 to 2007. In both males and females there was evidence that mortality decreased from 1993-1995 to 1999-2001 but increased from 1999-2001 to 20052007 (Table 1). The relative increase was approximately $28 \%$ in females and $17 \%$ in males.

Figure 3 shows the trends in agestandardised mortality rates for males and females in England and Wales from 1979 to 2007, based on death registrations, with epilepsy as the underlying cause, as recorded by the ONS. Over this period, there was evidence of a linear increase in deaths with epilepsy coded as the underlying cause. In males, the linear increase was $0.020 \quad 195 \%$ confidence interval $[\mathrm{Cl}]=0.014$ to 0.026$)$ per 100000 per year $(P<0.001)$; in females it was 0.009 


\begin{tabular}{|c|c|c|c|c|}
\hline & $\begin{array}{l}\text { All deaths in } \\
\text { persons with } \\
\text { prevalent epilepsy }\end{array}$ & $\begin{array}{l}\text { Age-standardised } \\
\text { mortality rate per } \\
100000 \text { registered } \\
\text { GPRD patients }(95 \% \mathrm{Cl})\end{array}$ & $\begin{array}{l}\text { Relative risk } \\
\text { (95\% CI) }\end{array}$ & $P$-value \\
\hline \multicolumn{5}{|c|}{ 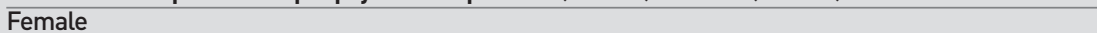 } \\
\hline 1993-1995 & 661 & 21 (19 to 22) & 1.12 (1.02 to 1.24$)$ & 0.023 \\
\hline 1996-1998 & 782 & 19 (17 to 20 ) & 1.02 (0.93 to 1.12 ) & 0.69 \\
\hline 1999-2001 & 1020 & 19 (18 to 21$)$ & Reference & \\
\hline $2002-2004$ & 1430 & 24 (22 to 25 ) & 1.23 (1.13 to 1.33 ) & $<0.001$ \\
\hline $2005-2007$ & 1573 & 25 (24 to 26 ) & 1.28 (1.18 to 1.39$)$ & $<0.001$ \\
\hline \multicolumn{5}{|l|}{ Male } \\
\hline 1993-1995 & 740 & 30 (27 to 32) & 1.16 (1.06 to 1.27 ) & 0.002 \\
\hline $1996-1998$ & 839 & 25 (24 to 27) & $1.00(0.92$ to 1.10$)$ & 0.92 \\
\hline 1999-2001 & 1127 & 26 (24 to 27) & Reference & \\
\hline 2002-2004 & 1488 & 29 (27 to 30 ) & 1.14 (1.05 to 1.23$)$ & 0.001 \\
\hline 2005-2007 & 1631 & 30 (29 to 32 ) & 1.17 (1.09 to 1.26$)$ & $<0.001$ \\
\hline
\end{tabular}

(95\% $\mathrm{Cl}=0.004$ to 0.014$)$ per 100000 per year $(P<0.001)$. When data were adjusted for the difference in coding between ICD-9 and ICD-10, there was no longer evidence of an increasing trend in females (trend per year $-0.001,95 \% \mathrm{Cl}=-0.007$ to $0.004, P=$ $0.65)$ but there was still evidence of an increasing trend in males (trend per year $0.021,95 \% \mathrm{Cl}=0.014$ to $0.028, P<0.001$ ). This sensitivity analysis suggests that the increase in epilepsy-coded deaths associated with ICD-10 may contribute to the rising trend in epilepsy mortality in females but not in males.

Table 2 shows the results of the nested case-control study. Results are presented for all years of death combined, and separately for years of death from 2004 to 2007. Deprivation quintile was not associated with mortality of persons with prevalent epilepsy, but recorded problems of alcohol misuse - although infrequent were strongly associated with mortality in males and females. Depression treated in the previous 12 months with antidepressant drugs, as well as one or more records of accidents or injuries in the preceding 12 months, was associated with increased risk of mortality.

Compared with participants who collected their last prescription for anticonvulsant drugs in the preceding
Figure 3. Mortality with epilepsy as underlying cause in general population of England and Wales (1979-2007). ${ }^{a}$

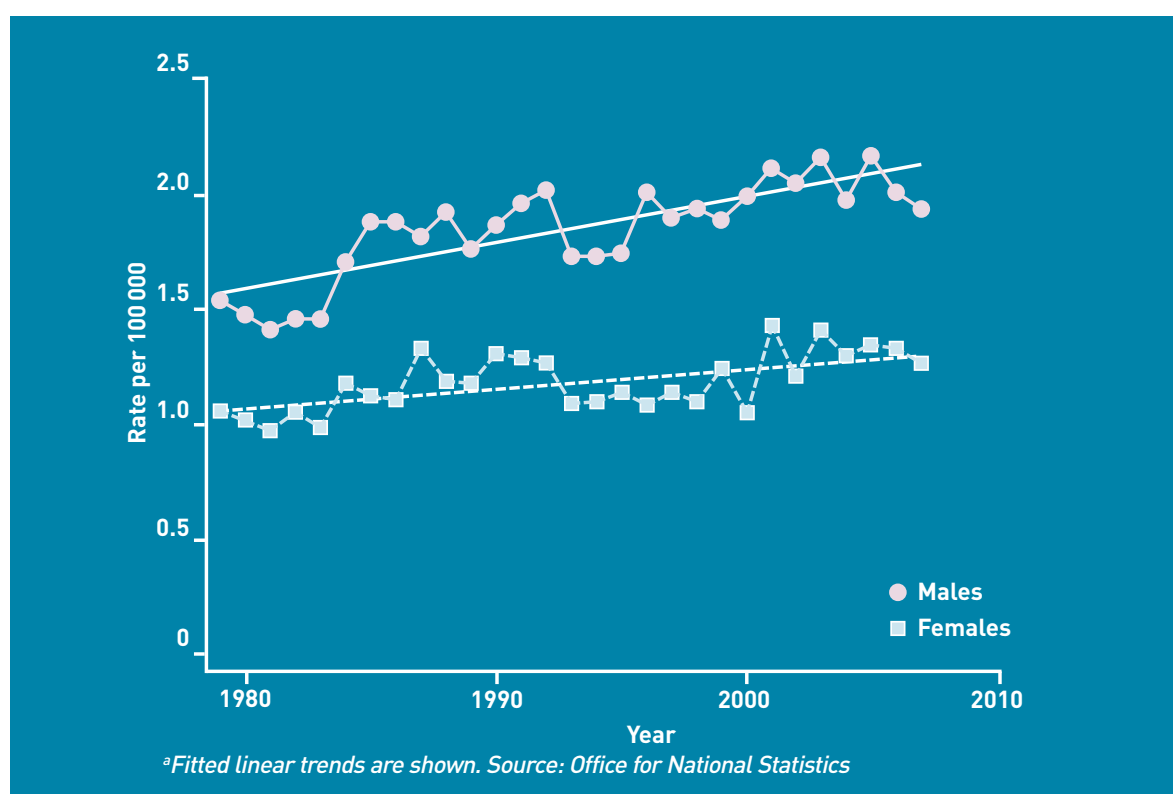




\section{Table 1. Variables associated with death of persons with prevalent epilepsya}

\begin{tabular}{|c|c|c|c|c|c|c|c|c|}
\hline \multicolumn{5}{|c|}{ All participants } & \multicolumn{4}{|c|}{ Cases with year of death $\geq 2004$} \\
\hline & Controls (\%) & Cases $(\%)$ & $\mathrm{OR}(95 \% \mathrm{Cl})$ & $P$-value & Controls (\%) & Cases $(\%)$ & OR $(95 \% \mathrm{CI})$ & $P$-value \\
\hline \multicolumn{9}{|c|}{ Deprivation quintile } \\
\hline 1 & $3236(18)$ & 1967 (18) & Reference & & 1653 (19) & $912(19)$ & Reference & \\
\hline 2 & 3184 (18) & $1879(17)$ & $1.00(0.92$ to 1.10$)$ & 0.94 & $1523(17)$ & $796(17)$ & 1.01 (0.89 to 1.15$)$ & 0.90 \\
\hline 3 & $3525(20)$ & 2075 (19) & 0.96 (0.89 to 1.05$)$ & 0.41 & $1728(19)$ & 959 (20) & 1.00 (0.88 to 1.13$)$ & 0.99 \\
\hline 4 & $3765(21)$ & $2119(20)$ & $0.93(0.86$ to 1.01$)$ & 0.10 & $1842(21)$ & $917(19)$ & $0.93(0.82$ to 1.06$)$ & 0.28 \\
\hline 5 & 4175 (23) & $2741(25)$ & 1.05 (0.97 to 1.14 ) & 0.27 & 2118 (24) & 1204 (25) & 1.03 (0.91 to1.15) & 0.68 \\
\hline NK & $13(0)$ & $16(0)$ & & & $7(0)$ & $11(0)$ & & \\
\hline No & 17790 (99) & 10585 (98) & Reference & & 8815 (99) & 4704 (98) & Reference & \\
\hline Yes & $108(1)$ & $212(2)$ & 2.96 (2.25 to 3.89$)$ & $<0.001$ & $56(1)$ & $95(2)$ & 2.71 (1.83 to 4.02 ) & $<0.001$ \\
\hline \multicolumn{9}{|l|}{ Depression } \\
\hline No & 16093 (90) & 9212 (85) & Reference & & 7865 (89) & 3970 (83) & Reference & \\
\hline Yes & $1805(10)$ & 1585 (15) & 1.39 (1.28 to 1.50$)$ & $<0.001$ & $1006(11)$ & $829(17)$ & 1.39 (1.25 to 1.56$)$ & $<0.001$ \\
\hline \multicolumn{9}{|c|}{ Injury/accident in previous 12 months } \\
\hline No & $16178(90)$ & $9151(85)$ & Reference & & 8094 (91) & $4125(86)$ & Reference & \\
\hline Yes & $1720(10)$ & $1646(15)$ & 1.41 (1.30 to 1.53$)$ & $<0.001$ & $777(9)$ & $674(14)$ & 1.36 (1.20 to 1.53$)$ & $<0.001$ \\
\hline$<90$ days & $10847(61)$ & $8250(76)$ & Reference & & 5250 (59) & 3724 (78) & Reference & \\
\hline $91-182$ days & $768(4)$ & $1117(10)$ & 1.83 (1.66 to 2.03$)$ & $<0.001$ & $343(4)$ & $429(9)$ & $1.72(1.47$ to 2.01$)$ & $<0.001$ \\
\hline $183-365$ days & $655(4)$ & $437(4)$ & 0.83 (0.72 to 0.94$)$ & 0.004 & 278 (3) & $169(4)$ & $0.77(0.63$ to 0.95$)$ & 0.015 \\
\hline$\geq 365$ days & $5019(28)$ & $908(8)$ & $0.24(0.22$ to 0.26$)$ & $<0.001$ & 2789 (31) & $426(9)$ & 0.20 (0.18 to 0.23$)$ & $<0.001$ \\
\hline Never & 609 (3) & $85(1)$ & & & $211(2)$ & $51(1)$ & & \\
\hline \multicolumn{9}{|c|}{ Recorded seizure free in previous 12 months } \\
\hline No & - & - & - & - & $7135(80)$ & 3771 (79) & Reference & \\
\hline Yes & - & - & - & - & $1736(20)$ & $1028(21)$ & 0.78 (0.71 to 0.86$)$ & $<0.001$ \\
\hline
\end{tabular}

$\geq 90$ days, participants who collected their last prescription 91-182 days (3-6 months) previously had a higher risk of mortality; those who had discontinued therapy for 26-12 months had a lower mortality risk. When data for participants with death dates in 2004 or later were analysed separately, adjusted analyses showed that a record of being seizure free in the preceding 12 months was associated with a lower risk of mortality. The unadjusted OR associated with being recorded seizure free was 1.14 $(95 \% \mathrm{Cl}=1.04$ to $1.24, P=0.005)$; however, after adjustment for the other variables included in the model, there was evidence of lower mortality associated with being seizure free. This indicates some degree of confounding with the other included risk factors.

\section{DISCUSSION}

\section{Summary}

The increasing recorded prevalence of epilepsy may indicate improved documentation of the condition in primary care records. The increase also suggests that, in this population, epilepsy cases are rising through diagnosis or practice recruitment at a faster rate than they are declining through death or end of registration. The present analyses using two separate data sources - GPRD data and national mortality registrations suggest that mortality in epilepsy is increasing. Data from primary care electronic patient records in the GPRD show that mortality increased during 19992007; however, earlier trends in the GPRD were inconsistent, with an initial increase being followed by an apparent decrease for reasons that are unclear. Death registrations reveal a longer-term trend of increasing numbers of deaths with epilepsy registered as the underlying cause from 1979 to the present. This increase in epilepsy mortality is in contrast to the substantial decline in all-cause mortality that is currently being observed in the general population.

The case-control study provides evidence of several distinct risk factors for mortality in epilepsy. The hypotheses tested were based on prior reviews of the epidemiological evidence. ${ }^{4,7}$ The risk factors for mortality identified in this analyses were recorded alcohol problems, a 'missed' prescription for anticonvulsant drugs, a history of injuries, or treatment for depression. 
The study did not quantify the duration of each anticonvulsant prescription, as experience shows that this cannot be estimated for a substantial proportion of prescriptions in the GPRD. However, treatment intervals of approximately 90 days are observed in other chronic illnesses in the GPRD. The present analyses suggested that participants whose last prescription was collected 3-6 months previously - which might be interpreted as a 'missed' prescription - were at increased risk of death; however, participants whose discontinuation of therapy was further in the past were at lower risk.

The authors did not have information on hospital prescriptions, and some participants who did not collect repeat prescriptions might have been attending hospital clinics. Nevertheless, the group of patients who do not collect repeat anticonvulsant prescriptions is important to identify in the clinical context. Problems of alcohol misuse were not frequently recorded but were strongly associated with mortality risk. In data obtained since the introduction of the QOF, participants who were recorded as seizure free were at lower mortality risk.

\section{Strengths and limitations}

The study findings are based on an analysis of patients coded by their GPs as having epilepsy. This definition differs from the one used in the QOF, which is only applied to people aged $\geq 18$ years, and includes individuals who have been diagnosed with epilepsy at some point in their life and currently receive anticonvulsant drug treatment. For this study it was wanted to include participants who may have discontinued anticonvulsant therapy; however, it is important to be aware that the definition of epilepsy may vary between GPs and it is likely that some apply the diagnosis more rigorously than others. Differences in apparent prevalence, based on the disease register, may, therefore, represent true differences in community prevalence or coding differences. Moreover, this analysis was unable to make comparisons between patients based on the severity of their epilepsy as GP records rarely contain coded data describing the frequency or duration of seizures.

This study was restricted to evaluating risk factors that are likely to be routinely coded into electronic patient records. There may be other important risk factors for which data were not available. Coding into electronic records may be associated with substantial misclassification, the general effect of which will be to reduce the magnitude of associations; however, when confounders are misclassified, the direction of bias may be difficult to anticipate. Regression models for data were fitted from two different time periods and tested several categories for some variables: although multiple testing was not adjusted for, readers may wish to view these results with an appropriate degree of caution.

Deprivation score quintile was not associated with outcomes but deprivation was retained in the model as it is generally associated with the quality of medical care and with mortality rates.

\section{Comparison with existing literature}

Evidence derived and recorded operationally using general practice computer systems can correspond closely with measurement using robust research techniques. ${ }^{12}$ The incidence of newly diagnosed epilepsy remained constant between 1993 and 2007, being 38 per 100000 in females and 40 per 100000 in males. The prevalence of epilepsy rose from 9 per 1000 in 1993 to 12 per 1000 in 2007 . It is not clear yet whether this is real trend or the product of more accurate or inflated recording. The level is higher than reported in an earlier study using the GPRD. ${ }^{13}$

\section{Implications for practice and research}

The GPRD offers a unique opportunity to examine conditions like epilepsy that have been hitherto neglected on a primary care population basis, and consider policies that might improve the quality of care and outcomes. After diagnosis by specialists, about two-thirds of people with epilepsy are managed by their GP, and most patients choose this type of management. ${ }^{14}$ However, until recently there has been no reimbursement for epilepsy care, and doctors in primary care expressed lack of confidence in epilepsy management. ${ }^{15}$ Only 15 out of 1000 points were allocated to reward epilepsy registration and monitoring; the National Institute for Health and Clinical Excellence and the Joint Epilepsy Council propose this be expanded. Death in epilepsy is more likely when 
seizures have continued to occur, there are problems with alcohol misuse, medication is not adhered to, injury has occurred in the previous year, or patients have been treated for depression. Recording of these risk factors needs to be linked up operationally in order to alert GPs to the need for 'stepup' care.

Failure to improve outcomes may be partially explained by a lack of integrated primary-secondary care pathways. The number of neurologists has trebled over the past decade, but secondary-tertiary links have traditionally been closer in neurology than links with primary care. Patients seen in primary care may be as likely to report seizures as patients seen in secondary care, ${ }^{16}$ suggesting a lack of access and triage. Poor epilepsy control is associated with higher levels of emergency epilepsy-related hospitalisation ${ }^{17}$ and three-quarters of patients are discharged with no further epilepsy care plan. ${ }^{18}$ Six out of seven of hospital admissions for epilepsy are through emergency and unplanned; ${ }^{19}$ this generates high secondary-care costs, with little benefit to patients.

The need for further research into causespecific mortality in epilepsy, the types of injuries observed in a population with epilepsy, and the utilisation and outcomes of different anticonvulsant therapies is acknowledged. The results of this study also have significant implications for policy and service development. Indicators can be created to reward primary care for offering each person with epilepsy a comprehensive care plan and, for those with recurrent seizures, a step-up plan. ${ }^{20}$ Enhancements to services need to be linked to epilepsy training for GPs and practice nurses.

The Royal College of Physicians is developing new models for neurologists who work in the districts; reducing epilepsy mortality should be a major aim. The QOF could incentivise GPs to refer back to specialists those people who do not remain seizure free. Recommendations could also include systems to alert when:

- prescriptions are not collected on time;

- accidents occur:

- depression is diagnosed and treated; and

- those with a dual alcohol-misuse diagnosis are referred for appropriate intervention.

Models for innovation in the service will require evaluation to provide evidence of improved quality and to demonstrate whether outcomes, particularly death, can be reduced. 


\section{REFERENCES}

1. Wheller L, Baker A, Griffiths C, Rooney C. Trends in avoidable mortality in England and Wales, 1993-2005. Health Stat Q 2007; (34): 6-25.

2. Office for National Statistics. Mortality statistics: general. review of the Register General on deaths in England and Wales, 2005. (URL is: http://www.statistics.gov.uk/downloads/theme_health/Dh1_38_2005/DH1_No 38.pdf. (accessed 4 Apr 2011).

3. Page A, Tobias M, Glover J, et al. Australian and New Zealand atlas of avoidable mortality. Adelaide: University of Adelaide, 2006.

4. Sander JW, Bell GS. Reducing mortality: an important aim of epilepsy management. J Neurol Neurosurg Psychiatry 2004; 75(3): 349-351.

5. Hanna JN, Black M, Sander JW, et al. National sentinel clinical audit of epilepsy-related death. London: The Stationery Office, 2002.

6. Doran T, Fullwood C, Gravelle H, et al. Pay-for-performance programs in family practices in the United Kingdom. New Eng J Med 2006; 355(4): 375-384

7. Monté CP, Arends JB, Tan IY, et al. Sudden unexpected death in epilepsy patients: risk factors a systematic review. Seizure 2006; 16(1): 1-7.

8. Herrett E, Thomas SL, Schoonen WM, et al. Validation and validity of diagnoses in the General Practice Research Database: a systematic review. Br J Clin Pharmacol 2010; 69(1): 4-14

9. British Medical Association and Royal Pharmaceutical Society of Great Britain. British National Formulary. London: BMJ Group and the Royal Pharmaceutical Society of Great Britain, 2011.

10. World Health Organisation. International Classification of Diseases. Ninth Revision. Geneva: World Health Organisation, 1979.

11. World Health Organisation. International Classification of Diseases. Tenth
Revision. Geneva: World Health Organisation, 2000.

12 Nazareth I, King M, Haines A, et al. Accuracy of diagnosis of psychosis on general practice computer system. BMJ 1993; 307(6895): 32-34.

13. Gaitatzis A, Purcell B, Carroll K, et al. Differences in the use of health services among people with and without epilepsy in the United Kingdom: socio-economic and disease-specific determinants. Epilepsy Res 2002; 50(3): 233-241.

14. Ridsdale L, Robins D, Fitzgerald A, et al. Epilepsy monitoring and advice recorded: general practitioners' views, current practice and patients' preferences. Br J Gen Pract 1996; 46(402): 11-14.

15. Thapar AK, Stott NCH, Richens A, Kerr M. Attitudes of GPs to the care of people with epilepsy. Fam Pract 1998; 15(5): 437-442.

16. Hart YM, Shorvon SD. The nature of epilepsy in the general population. II. Medical Care. Epilepsy Res 1995; 21(1): 51-58.

17. Shohet C, Yelloly J, Bingham P, Lyratzopolous G.The association between the quality of epilepsy management in primary care, general practice population deprivation status and epilepsy-related emergency hospitalisations. Seizure 2007; 6(4): 351-355.

18. Reuber M, Hattingh L, Goulding PJ. Epileptological emergencies in accident and emergency: a survey at St James's University Hospital, Leeds. Seizure 2000; 9(3): 216-220.

19. Majeed A, Bardsley M, Morgan D, et al. Cross sectional study of primary care groups in London: association of measures of socioeconomic and health status with hospital admission rates. BMJ 2000; 321(7268): 1057-1060.

20. Ridsdale $L$. The social causes of inequality in epilepsy and developing a rehabilitation strategy: a UK-based analysis. Epilepsia 2009; 50(10): 2175-2179. 